Pacific Journal of Mathematics

THE GENERALIZED M. RIESZ THEOREM AND

EARL Robert BERKSon AND THomas Alastair Gilled 


\title{
THE GENERALIZED M. RIESZ THEOREM AND TRANSFERENCE
}

\author{
EARl Berkson ANd T. A. Gillespie
}

\begin{abstract}
We use transference methods to give a new proof for Bochner's abstract generalization of the $M$. Riesz Theorem on conjugate harmonic functions. The proof makes direct use of the classical Hilbert transform, providing it with a structural role in the abstract setting. The norm of the classical Hilbert transform is thereby shown to be the least admissible bound in the abstract theorem. Analogous results are obtained for certain classical multiplier transforms related to the Hilbert transform.
\end{abstract}

1. Introduction. This note is concerned with the following theorem of Bochner, which provides in abstract settings a generalization of the classical Hilbert transform $\tilde{H}_{p}$ on $L^{p}(\mathbf{T})$, where $\mathbf{T}$ is the unit circle and $1<p<\infty$.

(1.1) Generalized M. Riesz Theorem. Let $G$ be a compact, connected, abelian group, and let $\geq$ be a linear ordering of its (additive) dual group $\hat{G}$. For $\gamma \in \hat{G}$, let $\operatorname{sgn} \gamma$ be $1,-1$, or 0 according as $\gamma>0, \gamma<0$, or $\gamma=0$, and put $h(\gamma)=-i \operatorname{sgn} \gamma$ for $\gamma \in \hat{G}$. For $1<p<\infty$, $h$ is an $L^{p}(G)$ multiplier, and

$$
\|h\|_{p, p} \leq A_{p},
$$

where $\|h\|_{p, p}$ is the multiplier norm of $h$, and $A_{p}$ is a constant depending only on $p$.

We give a new proof of the Generalized M. Riesz Theorem below. Our demonstration is achieved by an appropriate "transference" of the classical Hilbert transform $H_{p}$ defined on $L^{p}$ of the real line $\mathbf{R}$, and shows that $\left\|\tilde{H}_{p}\right\|$ is an admissible value for $A_{p}$ in (1.2). Thus $\left\|\tilde{H}_{p}\right\|$ is the minimum value for $A_{p}$.

Bochner's proof of the Generalized M. Riesz Theorem [1, Theorem 16] takes as its starting point the obvious fact that for trigonometric polynomials of "analytic type" (i.e., finite linear combinations of nonnegative characters) the map $f \mapsto \hat{f}(0)$ is an algebra homomorphism onto the complex field $\mathbf{C}$ ( $\hat{f}$ denotes the Fourier transform of $f$ with respect to the normalized Haar measure $\nu$ of $G$ ). From this it is immediate that the 
"analytic completion" $F$ of a real-valued trigonometric polynomial $f$ satisfies $\int_{G} F^{k} d \nu=[\hat{f}(0)]^{k}$ for each positive integer $k$. An argument which bears some close analogies with M. Riesz's original existence proof for $\tilde{H}_{p}$ (see [2, pp. 489-493] and [12, §7.21, especially equations (2), (4)]) then establishes the desired conclusion for $p$ an even integer. The case of arbitrary $p \geq 2$ is handled by interpolation using the Riesz Convexity Theorem. Dualizing completes the demonstration.

Later some elegant observations of Helson (see [7, proof of Theorem 2]) led to a different proof of the Generalized M. Riesz Theorem which makes more direct use of complex methods. Specifically, let $C_{a}(G)$ be the uniform closure of the trigonometric polynomials of analytic type. Thus $f \mapsto \hat{f}(0)$ is a homomorphism of $C_{a}(G)$. This fact and the Gelfand representation of $C_{a}(G)$, in conjunction with the operation of holomorphic functions on the elements of $C_{a}(G)$, show that if $f \in C_{a}(G)$, Ref $>0$, $0<p<\infty$, then $f^{p} \in C_{a}(G)$ and $\widehat{f^{p}}(0)=[\hat{f}(0)]^{p}$. Hence if $u$ is a positive trigonometric polynomial and $f$ is its "analytic completion," then $\int_{G} f^{p} d \nu$ $=\left(\int_{G} u d \nu\right)^{p}$. The demonstration can now be completed by integrating an appropriate inequality as in [10,8.7.4-(3)], or by following the classical proof of M. Riesz [12, page 148] mutatis mutandis.

In essence then the two previous proofs outlined above extend the classical proof to the abstract setting. While highly satisfactory from a descriptive standpoint, such a procedure, as in the case of the classical existence proof, does not involve or identify the least admissible value for $A_{p}$. The proof of the Generalized M. Riesz Theorem presented below in $\$ 2$ makes direct use of the classical Hilbert transform itself. This approach elucidates the structural role the Hilbert transform plays in the abstract setting, and, as mentioned above, identifies the least value of $A_{p}$ as $\left\|\tilde{H}_{p}\right\|$. We have been unable to find, in the literature or the folklore, a proof of the Generalized M. Riesz Theorem which has either of these two features. The medium used below to secure them is the transference method developed by R. Coifman and G. Weiss ([3], [4]) which makes it possible, under appropriate circumstances, to "transfer" the setting of a convolution operator (associated with a locally compact amenable group), while retaining estimates for the operator. As may be inferred from our earlier comments, this is the first application of transference to the Generalized M. Riesz Theorem. Outside of one transference theorem we use only elementary methods and standard tools of modern analysis. It might be worthwhile to observe that (apart from one's point of view concerning assumed facts about the Hilbert transform) no analytic function theory is employed in the proof of Theorem (2.1). 
In $\$ 3$ the paper closes with a systematic treatment showing that the multiplier norms of various classical multipliers associated with $\tilde{H}_{p}$ dominate those of their abstract analogues (see Theorem (3.8)). For this purpose it is necessary to utilize $L^{p}(\mathbf{R})$ multipliers which are not "normalized" in the sense of $[4$, p. 17], and so a slight adaptation of $[4$, Theorem 3.15] is noted in Proposition (3.3).

2. The transference proof. Throughout the remainder of this note we fix $p$ in the range $1<p<\infty$, and continue to denote by $\tilde{H}_{p}$ (resp., $H_{p}$ ) the Hilbert transform on $L^{p}(\mathbf{T})$ (resp., $L^{p}(\mathbf{R})$ ). It is well-known that $\left\|H_{p}\right\|=\left\|\tilde{H}_{p}\right\|$. (For example, the proof in $[12, \S 12.42]$ shows that $\left\|H_{p}\right\| \leq$ $\left\|\tilde{H}_{p}\right\|$, and the reverse inequality can be seen from [8, Proposition 3.3] or [11, Corollary 4.6].) For ease of reference we state our objective as a theorem.

(2.1) TheOREM. Let $G, \geq$, and $h$ be as in the hypotheses of Theorem (1.1). Then

(i) $h$ is an $L^{p}(G)$ multiplier, and

(ii) $\|h\|_{p, p} \leq\left\|\tilde{H}_{p}\right\|$.

Before proving Theorem (2.1), we establish two lemmas. Let $\mathbf{Z}$ denote the set of integers.

(2.2) LEMMA. In order that Theorem (2.1) be valid, it is sufficient that (2.1)-(i) and (2.1)-(ii) hold whenever $N$ is a positive integer, $G=\mathbf{T}^{N}$, and the additive group $\mathbf{Z}^{N}$ is endowed with any linear ordering.

Proof. Let $G_{0}$ be a compact, connected, abelian group, and let $\geq$ be an ordering of $\hat{G}_{0}$. In order to establish the lemma it is enough to show that if $\mathscr{A}$ is any finite subset of $\hat{G}_{0}$ containing a character distinct from the identity of $\hat{G}_{0}$, and if $c_{\gamma}, \gamma \in \mathscr{A}$, are complex constants, then

$$
\left\|Q_{2}\right\|_{L^{p}(G)} \leq\left\|\tilde{H}_{p}\right\|\left\|Q_{1}\right\|_{L^{p}(G)},
$$

where $Q_{1}=\sum_{\gamma \in \mathscr{A}} c_{\gamma} \gamma$, and $Q_{2}=\sum_{\gamma \in \mathscr{A}} h(\gamma) c_{\gamma} \gamma$. Let $\Lambda$ be the subgroup of $\hat{G}_{0}$ generated by $\mathscr{A}$, and let $K$ be the annihilator in $G_{0}$ of $\Lambda$. We identify $\left(G_{0} / K\right)$ with $\Lambda$ in the canonical way, and denote by $\tilde{\gamma}$ the character of $G_{0} / K$ corresponding to $\gamma \in \mathscr{A}$. Let $\tilde{Q}_{1}=\sum_{\gamma \in \mathscr{A}} c_{\gamma} \tilde{\gamma}$ and $\tilde{Q}_{2}=$ $\sum_{\gamma \in \mathscr{A}} h(\gamma) c_{\gamma} \tilde{\gamma}$. In view of the compactness of $G_{0}, K$, and $G_{0} / K$, their Haar measures of total mass 1 are normalized for the Fubini-like Weil formula $[9, \S 33]$. Since $K$ is the annihilator of $\Lambda \supseteq \mathscr{A}$, an obvious 
application of the Weil formula (needed here only for non-negative Baire functions) gives

$$
\left\|Q_{j}\right\|_{L^{p}\left(G_{0}\right)}=\left\|\tilde{Q}_{j}\right\|_{L^{p}\left(G_{0} / K\right)}, \quad \text { for } j=1,2 .
$$

So the proof of the lemma reduces to showing that

$$
\left\|\tilde{Q}_{2}\right\|_{L^{p}\left(G_{0} / K\right)} \leq\left\|\tilde{H}_{p}\right\|\left\|\tilde{Q}_{1}\right\|_{L^{p}\left(G_{0} / K\right)} .
$$

By the Fundamental Theorem on finitely generated Abelian groups, $\Lambda$ is a direct sum of $N$ non-zero cyclic subgroups, where $N$ is some positive integer. Since $\hat{G}_{0}$ is ordered, each of these subgroups has infinite order. Thus $\Lambda$ can be identified with $\mathbf{Z}^{N}$, and $G_{0} / K$ with $\mathbf{T}^{N}$. The ordering of $\hat{G}_{0}$ restricted to $\Lambda$ can be transported to $Z^{N}$, and it is now easy to see from the lemma's hypothesis for tori that (2.4) holds.

We are indebted to B. Reznick for suggestions used in the proof of the next lemma.

(2.5) LEMMA. Let $N$ be a positive integer, and let $\geq$ be any linear ordering of the additive group $\mathbf{Z}^{N}$. Denote by $C^{(+)}, C^{(-)}$the respective convex hulls in $\mathbf{R}^{N}$ of $\left\{z=\mathbf{Z}^{N}: z \geq 0\right\},\left\{z \in \mathbf{Z}^{N}: z<0\right\}$. Then $C^{(+)}$and $C^{(-)}$are disjoint.

Proof. Suppose, to the contrary, that $-\sum_{i=1}^{m} \alpha_{i} y_{i}=\sum_{j=1}^{n} \beta_{j} z_{j}$, where the $\alpha_{i}$ 's and $\beta_{j}$ 's are non-negative real numbers, $\sum_{i=1}^{m} \alpha_{i}=\sum_{j=1}^{n} \beta_{j}=1$, the $y_{i}$ 's are distinct positive lattice points, and the $z_{j}$ 's are distinct non-negative lattice points. Transposing gives $0=\sum_{i=1}^{m} \alpha_{i} y_{i}+\sum_{j \in J_{0}} \beta_{j} z_{j}$, where $J_{0}$ denotes $\left\{j: z_{j} \neq 0\right\}$. On dividing the last equation by $1+\sum_{j \in J_{0}} \beta_{j}$, we have that

$$
0=\sum_{k=1}^{s} \lambda_{k} w_{k}
$$

where $\lambda_{k}$ is a non-negative real number and $w_{k}$ a positive lattice point for $k=1, \ldots, s$, and $\sum_{k=1}^{s} \lambda_{k}=1$. The method of proof in Carathéodory's Convexity Theorem [6, page 36] shows that we can additionally assume that $s \geq 2$, and $w_{2}-w_{1}, \ldots, w_{s}-w_{1}$ are linearly independent in $\mathbf{R}^{N}$. It follows from (2.6), after replacing $\lambda_{1}$ by $\left(1-\sum_{k=2}^{s} \lambda_{k}\right)$, that

$$
-w_{1}=\sum_{k=2}^{s} \lambda_{k}\left(w_{k}-w_{1}\right) \text {. }
$$

On equating coordinates in $\mathbf{R}^{N}$ on both sides of (2.7), and taking account of the linear independence of $w_{2}-w_{1}, \ldots, w_{s}-w_{1}$, we see that $\left(\lambda_{2}, \ldots, \lambda_{s}\right)$ satisfies a linear system of equations with integer coefficients which has a unique solution in $\mathbf{R}^{s-1}$. It is elementary that this system must also be 
consistent over the rational field. It follows that $\lambda_{k}$ is a non-negative rational number for $k=1,2, \ldots, s$. Multiplication of (2.6) by a suitable positive integer now yields the absurd conclusion that 0 is a positive lattice point. This concludes the proof of Lemma (2.5).

We now take up the proof of Theorem (2.1). Let $N$ be a positive integer, and suppose that $\geq$ is a linear ordering of the additive group $\mathbf{Z}^{N}$. For $m \in \mathbf{Z}^{N}$, we denote by $\chi_{m}$ the corresponding character of $\mathbf{T}^{N}$. Let $E$ be a finite subset of $\mathbf{Z}^{N}$, and put $E^{+}=\{m \in E: m>0\}, E^{-}=\{m \in E$ : $m<0\}$. We suppose that $E^{+}$is non-void, $E^{+}=(-1) E^{-}$, and $0 \in E$. Let $a_{m}, m \in E$, be complex constants. In view of Lemma (2.2) it suffices, in order to establish Theorem (2.1), to show that

$$
\left\|P_{2}\right\|_{L^{p}\left(\mathbf{T}^{N}\right)} \leq\left\|\tilde{H}_{p}\right\|\left\|P_{1}\right\|_{L^{p}\left(\mathbf{T}^{N}\right)},
$$

where $P_{1}=\sum_{m \in E} a_{m} \chi_{m}, P_{2}=\sum_{m \in E} a_{m} h(m) \chi_{m}$. Let $K^{+}$(resp., $K^{-}$) be the convex hull in $\mathbf{R}^{N}$ of $E^{+}$(resp., $E^{-}$). Obviously $K^{+}$is non-void and compact, and $K^{-}=(-1) K^{+}$. By Lemma (2.5), $0 \notin K^{-}$. Hence $0 \notin K^{+}$. By the Basic Separation Theorem [5, Theorem V.2.10], there is a vector $\alpha \in \mathbf{R}^{N}$ such that $\alpha \cdot x>0$ for $x \in K^{+}$, where the dot is used to denote the inner product in $\mathbf{R}^{N}$. Since $K^{-}=(-1) K^{+}$, we have

$$
\alpha \cdot x>0 \text { for } x \in K^{+}, \quad \text { and } \quad \alpha \cdot x<0 \text { for } x \in K^{-} .
$$

Let $\alpha=\left(a_{1}, a_{2}, \ldots, a_{N}\right)$. For $t \in \mathbf{R}$ let $R_{t}^{(p)}$ (resp., $R_{t}^{(2)}$ ) be translation on $L^{p}\left(\mathbf{T}^{N}\right)\left(\right.$ resp., $\left.L^{2}\left(\mathbf{T}^{N}\right)\right)$ by $\left(\exp \left(i a_{1} t\right), \ldots, \exp \left(i a_{N} t\right)\right)$. Let $\mathscr{E}(\cdot)$ be the spectral measure of Stone's Theorem for the one-parameter unitary group $\left\{R_{t}^{(2)}\right\}, t \in \mathbf{R}$. With the aid of the Plancherel Theorem for $L^{2}\left(\mathbf{T}^{N}\right)$, it is easy to see that

$$
\text { for each Borel set } B \text { of } \mathbf{R}, \mathscr{E}(B) \text { is the multiplier }
$$

operator on $L^{2}\left(\mathbf{T}^{N}\right)$ corresponding to the characteristic function (relative to $\mathbf{Z}^{N}$ ) of $\left\{m \in \mathbf{Z}^{N}: \alpha \cdot m \in B\right\}$.

For now, we let $\lambda_{F}$ denote the characteristic function, relative to $\mathbf{Z}^{N}$, of a subset $F$. We now represent each $t \in \mathbf{R}$ by the translation of the group $\mathbf{T}^{N}$ corresponding to $\left(\exp \left(-i a_{1} t\right), \ldots, \exp \left(-i a_{N} t\right)\right)$. Thus the transference context of $[4, \S 3]$ is realized. We next apply [4, Theorem 3.15] to the normalized $L^{p}(\mathbf{R})$ multiplier $c$, where $c(y)=-i \operatorname{sgn} y$ for $y \in \mathbf{R}$. It follows, with the aid of (2.10), that

$$
\phi \equiv i \lambda_{\left\{m \in \mathbf{Z}^{N}: \boldsymbol{\alpha} \cdot m<0\right\}}-i \lambda_{\left\{m \in \mathbf{Z}^{N}: \boldsymbol{\alpha} \cdot m>0\right\}}
$$

is an $L^{p}\left(\mathbf{T}^{N}\right)$ multiplier, whose multiplier norm $\|\phi\|_{p, p}$ does not exceed $\left\|H_{p}\right\|\left(=\left\|\tilde{H}_{p}\right\|\right)$. 
Let $T_{\phi}$ be the multiplier operator on $L^{p}\left(\mathbf{T}^{N}\right)$ corresponding to $\phi$. Making use of (2.9), we see readily that $T_{\phi}\left(P_{1}\right)=P_{2}$. It is now evident that (2.8) holds, and the proof of Theorem (2.1) is complete.

3. Bounds for abstract multipliers associated with $h$. An obviously equivalent reformulation of the conclusion in Theorem (1.1) asserts that the characteristic function of $\{\gamma \in \hat{G}: \gamma \geq 0\}$ is an $L^{p}(G)$ multiplier, and the norm of the corresponding operator, "analytic projection (relative to $\geq$ ) for $L^{p}(G)$," is bounded by a constant $B_{p}$ depending only on $p$. A particular consequence of the main result of this section (Theorem (3.8)) is that we can take $B_{p}$ to be the norm of analytic projection for $L^{p}(\mathbf{T})$. Theorem (3.8) systematically provides a similar conclusion for any $L^{p}(G)$ multiplier which has constant values $\mu_{1}, \mu_{2}$ on $\{\gamma \in \hat{G}: \gamma>0\}$ and $\{\gamma \in \hat{G}: \gamma<0\}$, respectively, and whose value at 0 is on the segment joining $\mu_{1}$ and $\mu_{2}$. These multipliers are treated by transferring the corresponding $L^{p}(\mathbf{R})$ multipliers. Although the latter are not "normalized" in the sense of $[4, \S 3]$, a slight variant of $[4$, Theorem 3.15], observed in Proposition (3.3) below, renders them transferable. The classical estimate in Proposition (3.5) is used to complete the stage setting.

If $\mathscr{G}$ is a locally compact abelian group with dual group $\Gamma$, we denote the space of $L^{p}(\mathscr{G})$ multipliers by $M_{p}(\Gamma)$. To avoid confusion it will be convenient henceforth to symbolize the $L^{p}(\mathscr{G})$ multiplier norm of $\psi \in$ $M_{p}(\Gamma)$ by $\|\psi\|_{M_{p}(\Gamma)}$. For ease of reference we next state two well-known lemmas, the first a consequence of Parseval's formula and Hölder's inequality. We omit their proofs, which are straightforward, standard arguments.

(3.1) Lemma. Let $\mathscr{G}, \Gamma$ be as described above. If $\phi \in L^{1}(\Gamma)$ and $\psi \in M_{p}(\Gamma)$, then the convolution $\phi * \psi \in M_{p}(\Gamma)$, and

$$
\|\phi * \psi\|_{M_{p}(\Gamma)} \leq\|\phi\|_{L^{1}(\Gamma)}\|\psi\|_{M_{p}(\Gamma)} .
$$

(3.2) Lemma. Suppose $0 \leq \tau \leq 1$, and for $n=1,2, \ldots$, let $K_{n}^{(\tau)} \in L^{1}(\mathbf{R})$ satisfy:

(i) $K_{n}^{(\tau)} \geq 0$;

(ii) $K_{n}^{(\tau)}(x)=0$ for $|x|>n^{-1}$;

(iii) $\int_{0}^{+\infty} K_{n}^{(\tau)}(x) d x=1-\tau$;

(iv) $\int_{-\infty}^{0} K_{n}^{(\tau)}(x) d x=\tau$.

Then if $\phi: \mathbf{R} \rightarrow \mathbf{C}$ is bounded and measurable, $x_{0} \in \mathbf{R}$, and $\lim _{x \rightarrow x_{0}^{+}} \phi(x)=\mu_{1}, \lim _{x \rightarrow x_{0}^{-}} \phi(x)=\mu_{2}$, we have

$$
\lim _{n}\left(K_{n}^{(\tau)} * \phi\right)\left(x_{0}\right)=\tau \mu_{1}+(1-\tau) \mu_{2} \text {. }
$$


We next take up a slight extension of the transference theorem for multipliers [4, Theorem 3.15]. We follow the setting and terminology of [4, $\S 3]$. In particular, $\mathscr{G}$ is $\sigma$-compact, and $S$ is a representation of $\mathscr{G}$ consisting of measure-preserving transformations of a $\sigma$-finite measure space $\mathscr{M}$. $R_{u}^{(p)} f=f \circ S_{-u}$ for $u \in \mathscr{G}, f \in L^{p}(\mathscr{M}) . \mathscr{E}_{0}(\cdot)$ is the spectral measure of Stone's Theorem for the unitary representation $g \mapsto R_{g}^{(2)}$. We denote the set of positive integers by $\mathbf{N}$.

(3.3) Proposition. Let $m$ be a bounded measurable function on $\Gamma$. Suppose $\left\{m_{n}\right\}$ is a sequence of normalized functions in $M_{p}(\Gamma)$ such that $m_{n} \rightarrow m$ pointwise on $\Gamma, \sup \left\{\left|m_{n}(\gamma)\right|: \quad n \in \mathbf{N}, \quad \gamma \in \Gamma\right\}<\infty$, and $\sup _{n}\left\|m_{n}\right\|_{M_{p}(\Gamma)}<\infty$. Then

$$
\left(\int_{\Gamma} m d \mathscr{E}_{0}\right) \mid L^{2}(\mathscr{M}) \cap L^{p}(\mathscr{M})
$$

extends to a bounded linear mapping $Y_{m}$ of $L^{p}(\mathscr{M})$ into itself satisfying $\left\|Y_{m}\right\| \leq \sup _{n}\left\|m_{n}\right\|_{M_{p}(\Gamma)}$.

Proof. Let $q$ be the index conjugate to $p$. For each positive integer $n$, let $Y_{m_{n}}$ be the extension to $L^{p}(\mathscr{M})$ of $\left(\int_{\Gamma} m_{n} d \mathscr{E}_{0}\right) \mid L^{2}(\mathscr{M}) \cap L^{p}(\mathscr{M})$ as in [4, Theorem 3.15]. Thus $\left\|Y_{m_{n}}\right\| \leq\left\|m_{n}\right\|_{M_{p}(\Gamma)}$. Since a normalized function is Borel measurable, so is $m$. For $f \in L^{2}(\mathscr{M}) \cap L^{p}(\mathscr{M}), g \in L^{2}(\mathscr{M}) \cap$ $L^{q}(\mathscr{M})$,

$$
\left(Y_{m_{n}} f, g\right)=\int_{\Gamma} m_{n}(\gamma) d\left(\mathscr{E}_{0}(\gamma) f, g\right) .
$$

It follows from (3.4) and bounded convergence that

$$
\left|\int_{\Gamma} m(\gamma) d\left(\mathscr{E}_{0}(\gamma) f, g\right)\right| \leq\|f\|_{p}\|g\|_{q} \sup _{n}\left\|m_{n}\right\|_{M_{p}(\Gamma)} .
$$

The desired conclusion is now apparent.

REMARK. It is a well-known consequence of Parseval's formula that the hypotheses of Proposition (3.3) (without the requirement that each $m_{n}$ is normalized or the assumption that $\left.\sup \left\{\left|m_{n}(\gamma)\right|: n \in \mathbf{N}, \gamma \in \Gamma\right\}<\infty\right)$ imply that $m \in M_{p}(\Gamma)$, and $\|m\|_{M_{p}(\Gamma)} \leq \sup _{n}\left\|m_{n}\right\|_{M_{p}(\Gamma)}$. However, we shall not require the presence of these additional conclusions in Proposition (3.3).

(3.5) Proposition. Let $\zeta_{1}, \zeta_{2}, \eta \in \mathbf{C}$. Then

$$
\left\|\zeta_{1} I+\zeta_{2} H_{p}\right\| \leq\left\|\zeta_{1} \tilde{I}+\zeta_{2} \tilde{H}_{p}+\eta \tilde{P}_{0}\right\|,
$$

where $\tilde{I}$ denotes the identity operator of $L^{p}(\mathbf{T})$, and for each $f \in L^{p}(\mathbf{T}), \tilde{P}_{0} f$ is the constant function $\hat{f}(0)$ on $\mathbf{T}$. 
Proof. We follow the notation and methods of [12, pp. 317, 318]. Fix $f \in L^{p}(\mathbf{R})$ and put

$$
\begin{gathered}
g_{n}(x)=\frac{1}{2 \pi n} \int_{-n \pi}^{n \pi} f(t) \cot \frac{x-t}{2 n} d t, \\
\eta_{n}=\frac{\eta}{2 \pi n} \int_{-n \pi}^{n \pi} f(t) d t,
\end{gathered}
$$

for $n \in \mathbf{N}$. Then $\left|\eta_{n}\right| \leq|\eta|(2 \pi n)^{-1}\|f\|_{L^{p}(\mathbf{R})}(2 \pi n)^{1 / q}$, where $q$ is the index conjugate to $p$. Thus $\eta_{n} \rightarrow 0$. As shown in [12] $g_{n} \rightarrow H_{p} f$ a.e. on $\mathbf{R}$. Put $\phi_{n}(x)=f(n x)$ for $|x|<\pi$. Then it is easy to see by a suitable change of variables that

$$
\begin{aligned}
{\left[\left(\zeta_{1} \tilde{I}+\zeta_{2} \tilde{H}_{p}+\eta \tilde{P}_{0}\right) \phi_{n}\right](x)=\zeta_{1} f(n x)+\zeta_{2} g_{n}(n x)+\eta_{n}, } & \text { a.e. } \\
& \text { for }|x|<\pi .
\end{aligned}
$$

Hence

$$
\begin{array}{r}
{\left[(2 \pi)^{-1} \int_{-\pi}^{\pi}\left|\zeta_{1} f(n x)+\zeta_{2} g_{n}(n x)+\eta_{n}\right|^{p} d x\right]^{1 / p}} \\
\leq\left\|\zeta_{1} \tilde{I}+\zeta_{2} \tilde{H}_{p}+\eta \tilde{P}_{0}\right\|\left\|\phi_{n}\right\|_{L^{p}(\mathbf{T})} .
\end{array}
$$

Since $\left\|\phi_{n}\right\|_{L^{p}(\mathbf{T})} \leq(2 \pi n)^{-1 / p}\|f\|_{L^{p}(\mathbf{R})}$, a change of variables in (3.6) gives

$$
\begin{aligned}
& \left\{\int_{-n \pi}^{n \pi}\left|\zeta_{1} f(t)+\zeta_{2} g_{n}(t)+\eta_{n}\right|^{p} d t\right\}^{1 / p} \\
& \leq\left\|\zeta_{1} \tilde{I}+\zeta_{2} \tilde{H}_{p}+\eta \tilde{P}_{0}\right\|\|f\|_{L^{p}(\mathbf{R})} .
\end{aligned}
$$

Application of Fatou's Lemma to (3.7) gives the desired conclusion.

If $F$ is a subset of the dual group $\Gamma$ of a locally compact abelian group $\mathscr{G}$, we shall denote the characteristic function of $F$ relative to $\Gamma$ by $\lambda_{F}$. If $\Gamma$ is an ordered group, we shall, when convenient, let $\lambda_{+}$(resp., $\lambda_{-}$) symbolize the characteristic function of $\{\gamma \in \Gamma: \gamma>0\}$ (resp., $\{\gamma \in \Gamma$ : $\gamma<0\}$ ). If $\Gamma$ is $\mathbf{R}$ or $\mathbf{Z}$, the natural ordering of $\Gamma$ will be understood when the notation $\lambda_{+}, \lambda_{-}$is employed.

(3.8) Theorem. Let $G, \hat{G}$, and $\geq$ be as in the hypotheses of Theorem (1.1). Let $\mu_{1}, \mu_{2} \in \mathbf{C}$, and let $\mu$ belong to the line segment joining $\mu_{1}$ and $\mu_{2}$. Then the function $\Psi_{\mu_{1}, \mu_{2}, \mu}: \hat{G} \rightarrow \mathbf{C}$ given by

$$
\Psi_{\mu_{1}, \mu_{2}, \mu}=\mu_{1} \lambda_{+}+\mu_{2} \lambda_{-}+\mu \lambda_{\{0\}}
$$

is an $L^{p}(G)$ multiplier $(1<p<\infty)$, and

$$
\left\|\Psi_{\mu_{1}, \mu_{2}, \mu}\right\|_{M_{p}(\hat{G})} \leq C_{p, \mu_{1}, \mu_{2}},
$$


where $C_{p, \mu_{1}, \mu_{2}}=\left\|\mu_{1} \lambda_{+}+\mu_{2} \lambda_{-}\right\|_{M_{p}(\mathbf{R})}$. The particular constant $C_{p, \mu_{1}, \mu_{2}}$ is best possible as $G$ and $\geq$ vary, and is attained for $G=\mathbf{T}$ ( with the usual ordering of $\mathbf{Z}$ ).

Proof. To establish that $\Psi_{\mu_{1}, \mu_{2}, \mu} \in M_{p}(\hat{G})$ and that (3.9) is valid, let $N, \geq, E, E^{+}, E^{-},\left\{a_{m}\right\}, m \in E$, be as described just prior to (2.8). Let $s_{1}, s_{2}$ be the trigonometric polynomials on $\mathbf{T}^{N}$ given, respectively, by

$$
s_{1}=\sum_{m \in E} a_{m} \chi_{m}, \quad s_{2}=\sum_{m \in E} \Psi_{\mu_{1}, \mu_{2}, \mu}(m) a_{m} \chi_{m} .
$$

As in $\S 2$, it suffices to show that

$$
\left\|s_{2}\right\|_{L^{p}\left(\mathbf{T}^{N}\right)} \leq C_{p, \mu_{1}, \mu_{2}}\left\|s_{1}\right\|_{L^{p}\left(\mathbf{T}^{N}\right)} .
$$

Let $K^{+}$(resp., $K^{-}$) be the convex hull in $\mathbf{R}^{N}$ of $E^{+}$(resp., $E^{-}$). As in the proof of Theorem (2.1), there is a vector $\alpha \in \mathbf{R}^{N}$ such that (2.9) holds in the present circumstances; moreover, with $\left\{R_{t}^{(2)}\right\}, \mathscr{E}(\cdot)$ as before, (2.10) persists. Let $\mu=\tau \mu_{1}+(1-\tau) \mu_{2}$, where $0 \leq \tau \leq 1$, and pick a sequence $\left\{K_{n}^{(\tau)}\right\}$ as in Lemma (3.2) with each $K_{n}^{(\tau)}$ continuous. Let $W: \mathbf{R} \rightarrow \mathbf{C}$ be given by

$$
W=\mu_{1} \lambda_{(0,+\infty)}+\mu_{2} \lambda_{(-\infty, 0)}+\mu \lambda_{\{0\}},
$$

and put $W_{n}=K_{n}^{(\tau)} * W$. Since $W \in M_{p}(\mathbf{R})$, it follows from Lemma (3.1) that each $W_{n} \in M_{p}(\mathbf{R})$, and for all $n$,

$$
\left\|W_{n}\right\|_{M_{p}(\mathbf{R})} \leq\|W\|_{M_{p}(\mathbf{R})}=C_{p, \mu_{1}, \mu_{2}} .
$$

Each $W_{n}$ is continuous, hence normalized. By Lemma (3.2) $W_{n} \rightarrow W$ pointwise on R. From Proposition (3.3) we see with the aid of (3.11) and (2.10) that the function $\Phi$ on $\mathbf{Z}^{N}$ given by

$$
\Phi=\mu_{1} \lambda_{\left\{k \in \mathbf{Z}^{N}: \alpha \cdot k>0\right\}}+\mu_{2} \lambda_{\left\{k \in \mathbf{Z}^{N}: \alpha \cdot k<0\right\}}+\mu \lambda_{\left\{k \in \mathbf{Z}^{N}: \alpha \cdot k=0\right\}}
$$

is an $L^{p}\left(\mathbf{T}^{N}\right)$ multiplier, and $\|\Phi\|_{M_{p}\left(\mathbf{Z}^{N}\right)} \leq C_{p, \mu_{1}, \mu_{2}}$. From (2.9) $\Phi(m)=$ $\Psi_{\mu_{1}, \mu_{2}, \mu}(m)$ for $m \in E$. It is now clear that the multiplier operator on $L^{p}\left(\mathbf{T}^{N}\right)$ corresponding to $\Phi$ transforms $s_{1}$ into $s_{2}$, and (3.10) follows at once. Thus (3.9) is established in the general case.

To verify the last assertion of the theorem, we first apply (3.9) to the special case when $G=\mathbf{T}$ and $\mathbf{Z}$ has its usual ordering. This gives

$$
\left\|\mu_{1} \lambda_{+}+\mu_{2} \lambda_{-}+\mu \lambda_{\{0\}}\right\|_{M_{p}(\mathbf{Z})} \leq\left\|\mu_{1} \lambda_{+}+\mu_{2} \lambda_{-}\right\|_{M_{p}(\mathbf{R})} \text {. }
$$

Next we take $\zeta_{1}, \zeta_{2}, \eta$ in Proposition (3.5) to be, respectively, $\left(\mu_{1}+\mu_{2}\right) / 2$, $i\left(\mu_{1}-\mu_{2}\right) / 2,\left[\mu-2^{-1}\left(\mu_{1}+\mu_{2}\right)\right]$. This gives the reverse inequality to (3.12). Thus

$$
\left\|\Psi_{\mu_{1}, \mu_{2}, \mu}\right\|_{M_{p}(\mathbf{Z})}=C_{p, \mu_{1}, \mu_{2}} .
$$

This completes the proof of the theorem. 
(3.13) Corollary. Suppose $1<p<\infty$ and $\mu_{1}, \mu_{2} \in$ C. Let $\mu$ be a point on the line segment joining $\mu_{1}$ and $\mu_{2}$. Then

$$
\left\|\mu_{1} \lambda_{+}+\mu_{2} \lambda_{-}+\mu \lambda_{\{0\}}\right\|_{M_{p}(\mathbf{Z})}=\left\|\mu_{1} \lambda_{+}+\mu_{2} \lambda_{-}\right\|_{M_{p}(\mathbf{R})} .
$$

\section{REFERENCES}

[1] S. Bochner, Additive set functions on groups, Annals of Math., 40 (1939), 769-799.

[2] M. L. Cartwright, Manuscripts of Hardy, Littlewood, Marcel Riesz, and Titchmarsh, Bull. London Math. Soc., 14 (1982), 472-532.

[3] R. Coifman and G. Weiss, Operators associated with representations of amenable groups, singular integrals induced by ergodic flows, the rotation method, and multipliers, Studia Math., 47 (1973), 285-303.

[4] , Transference methods in analysis, Regional Conference Series in Math., No. 31, Amer. Math. Soc., Providence, 1977.

[5] N. Dunford and J. T. Schwartz, Linear operators-Part I: General theory, Pure and Applied Math., vol. 7, Interscience, New York, 1958.

[6] H. G. Eggleston, Convexity, Cambridge Tracts in Math. and Math. Physics, No. 47, Cambridge Univ. Press, 1958.

[7] H. Helson, Conjugate series and a theorem of Paley, Pacific J. Math., 8 (1958), 437-446.

[8] K. de Leeuw, On $L_{p}$ multipliers, Annals of Math., 81 (1965), 364-379.

[9] L. Loomis, An Introduction to Abstract Harmonic Analysis, Van Nostrand, New York, 1953.

[10] W. Rudin, Fourier analysis on groups, Interscience Tracts in Pure and Applied Math., No. 12, Interscience, New York, 1962.

[11] S. Saeki, Translation invariant operators on groups, Tôhoku Math. J., 22 (1970), 409-419.

[12] A. Zygmund, Trigonometrical series, Dover, New York, 1955.

Received April 12, 1984. The work of the first author was supported by the National Science Foundation (U.S.A.). The work of the second author was supported by the Science and Engineering Research Council (U.K.), and was undertaken while he was visiting Indiana University.

UNIVERSITY OF ILLINOIS

URBANA, IL 61801

AND

UNIVERSITY OF EDINBURGH

EDINBURGH EH9 3JZ, SCOTLAND 


\section{PACIFIC JOURNAL OF MATHEMATICS EDITORS}

\author{
V. S. VARADARAJAN (Managing Editor) \\ University of California \\ Los Angeles, CA 90024 \\ Charles R. DePrima \\ California Institute of Technology \\ Pasadena, CA 91125 \\ R. FINN \\ Stanford University \\ Stanford, CA 94305
}

\author{
HeRmanN FlaschKa \\ University of Arizona \\ Tucson, AZ 857.21
}

RAMESH A. GANGOLli

University of Washington

Seattle, WA 98195

ROBION KIRBY

University of California

Berkeley, CA 94720
C. C. MOORE

University of California

Berkeley, CA 94720

H. SAMELSON

Stanford University

Stanford, CA 94305

HAROLD STARK

University of California, San Diego

La Jolla, CA 92093

\section{ASSOCIATE EDITORS}
R. ARENS
E. F. BECKENBACH
B. H. NeumanN
F. WOLF
K. YoSHIDA
(1906-1982)

\section{SUPPORTING INSTITUTIONS}

\begin{abstract}
UNIVERSITY OF ARIZONA
UNIVERSITY OF BRITISH COLUMBIA

CALIFORNIA INSTITUTE OF TECHNOLOGY

UNIVERSITY OF CALIFORNIA

MONTANA STATE UNIVERSITY

UNIVERSITY OF NEVADA, RENO

NEW MEXICO STATE UNIVERSITY

OREGON STATE UNIVERSITY
\end{abstract}

\author{
UNIVERSITY OF OREGON \\ UNIVERSITY OF SOUTHERN CALIFORNIA \\ STANFORD UNIVERSITY \\ UNIVERSITY OF HAWAII \\ UNIVERSITY OF TOKYO \\ UNIVERSITY OF UTAH \\ WASHINGTON STATE UNIVERSITY \\ UNIVERSITY OF WASHINGTON
}

The Supporting Institutions listed above contribute to the cost of publication of this Journal, but they are not owners or publishers and have no responsibility for its content or policies.

Mathematical papers intended for publication in the Pacific Journal of Mathematics should be in typed form or offset-reproduced (not dittoed), double spaced with large margins. Please do not use built up fractions in the text of the manuscript. However, you may use them in the displayed equations. Underline Greek letters in red, German in green, and script in blue. The first paragraph must be capable of being used separately as a synopsis of the entire paper. In particular it should contain no bibliographic references. Please propose a heading for the odd numbered pages of less than 35 characters. Manuscripts, in triplicate, may be sent to any one of the editors. Please classify according to the scheme of Math. Reviews, Index to Vol. 39. Supply name and address of author to whom proofs should be sent. All other communications should be addressed to the managing editor, or Elaine Barth, University of California, Los Angeles, California 90024.

There are page-charges associated with articles appearing in the Pacific Journal of Mathematics. These charges are expected to be paid by the author's University, Government Agency or Company. If the author or authors do not have access to such Institutional support these charges are waived. Single authors will receive 50 free reprints; joint authors will receive a total of 100 free reprints. Additional copies may be obtained at cost in multiples of 50 .

The Pacific Journal of Mathematics is issued monthly as of January 1966. Regular subscription rate: $\$ 190.00$ a year (5 Vols., 10 issues). Special rate: $\$ 66.00$ a year to individual members of supporting institutions.

Subscriptions, orders for numbers issued in the last three calendar years, and changes of address should be sent to Pacific Journal of Mathematics, P.O. Box 969, Carmel Valley, CA 93924, U.S.A. Old back numbers obtainable from Kraus Periodicals Co., Route 100, Millwood, NY 10546.

The Pacific Journal of Mathematics at P.O. Box 969, Carmel Valley, CA 93924 (ISSN 0030-8730) publishes 5 volumes per year. Application to mail at Second-class postage rates is pending at Carmel Valley, California, and additional mailing offices. Postmaster: Send address changes to Pacific Journal of Mathematics, P.O. Box 969, Carmel Valley, CA 93924.

PUBLISHED BY PACIFIC JOURNAL OF MATHEMATICS, A NON-PROFIT CORPORATION

Copyright $\odot 1985$ by Pacific Journal of Mathematics 


\section{Pacific Journal of Mathematics}

Vol. 120, No. $2 \quad$ October, 1985

Philip Marshall Anselone and Mike Treuden, Regular operator

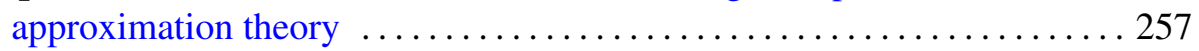

Giuseppe Baccella, Semiprime $\aleph-Q F 3$ rings $\ldots \ldots \ldots \ldots \ldots \ldots \ldots \ldots . \ldots \ldots$

Earl Robert Berkson and Thomas Alastair Gillespie, The generalized M.

Riesz theorem and transference $\ldots \ldots \ldots \ldots \ldots \ldots \ldots \ldots . \ldots 279$

Joachim Boidol, A Galois-correspondence for general locally compact

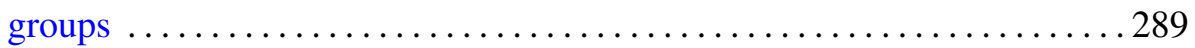

Joseph Eugene D'Atri, Josef Dorfmeister and Yan Da Zhao, The isotropy

representation for homogeneous Siegel domains ............... 295

C. Debiève, On Banach spaces having a Radon-Nikodým dual

Michael Aaron Freedman, Existence of strong solutions to singular

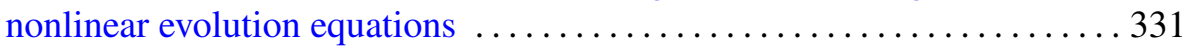

Francisco Jose Freniche, Grothendieck locally convex spaces of continuous

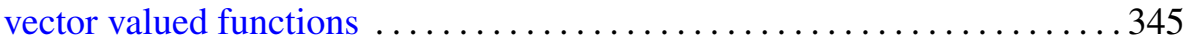

Hans-Peter Künzi and Peter Fletcher, Extension properties induced by complete quasi-uniformities . ............................ 357

Takaŝi Kusano, Charles Andrew Swanson and Hiroyuki Usami, Pairs of

positive solutions of quasilinear elliptic equations in exterior domains . . 385

Angel Rafael Larotonda and Ignacio Zalduendo, Spectral sets as Banach

manifolds

J. Martínez-Maurica and C. Pérez García, A new approach to the

Kreı̆n-Milman theorem

Christian Pommerenke, On the boundary continuity of conformal maps . . . 423

M. V. Subba Rao, Some Rogers-Ramanujan type partition theorems

Stephen Edwin Wilson, Bicontactual regular maps .........

Jaap C. S. P. van der Woude, Characterizations of (H)PI extensions

Kichoon Yang, Deformation of submanifolds of real projective space

Subhashis Nag, Errata: "On the holomorphy of maps from a complex to a

real manifold" 